P99 (continued)

Objective: To investigate whether offering a vegetable appetizer in a restaurant setting will impact children's vegetable consumption at a meal and whether marketing cues during the appetizer course can further increase vegetable consumption.

Study Design, Setting, Participants, Intervention: Families with at least one child (4-12 years) were recruited from university listservs to attend three dinners serving the same meal (chicken strips, french fries, carrot sticks, ranch dressing, and milk). For Meal 1 children were served all foods simultaneously (control condition). During Meals 2 and 3, the vegetable (carrot sticks) was offered as an appetizer. Meal 3 included marketing prompts highlighting carrots during the appetizer course. Foods offered to children were weighed pre- and post-meal to determine consumption.

Outcome Measures and Analysis: Repeated measures analysis of variance was conducted to determine the effects on carrot consumption (g) with main effects of Meal condition and age (4-7 and 8-12 years).

Results: All families $(n=19)$ participated in three dinners at a university-based restaurant $(n=34$ children, $n=33$ parents). There was a significant effect of Meal condition on carrot consumption $[\mathrm{F}(2,31)=3.50, P=.036]$. Children consumed significantly more carrots (g) during the appetizer conditions compared to the control condition $(13.5 \mathrm{~g}+36.9, P=.04)$. The addition of marketing prompts at Meal 3 did not further increase carrot consumption, $P=.95$. There was a significant main effect for age $[\mathrm{F}(2,31)=3.80, P=.028]$ with younger children demonstrating larger increases in intake $(\overline{\mathrm{x}}=18.7 \mathrm{~g})$ compared to older children $(\overline{\mathrm{x}}=9.0 \mathrm{~g})$.

Conclusions and Impolications: These pilot data suggest that offering a vegetable appetizer may be a feasible strategy to increase children's consumption of vegetables in the restaurant setting, especially for younger children.

Funding: Colorado State University, College of Health and Human Sciences.

\section{P100 Organizational Readiness for Implementing a Nutrition Curriculum in Early Care and Education}

Taren Swindle,PhD, TSwindle@uams.edu, University of Arkansas for Medical Sciences, 521 Jack Stephens Drive \#530, Little Rock, AR 72205-7199;

Leanne Whiteside-Mansell, EdD; Danya Johnson, BS

Background (Background, Rationale, Prior Research, and/or Theory): Preschool educational standards increasingly emphasizes nutrition education as a promising obesity prevention strategy. Success of implementation of new curricula likely vary based on the readiness of the context. An assessment of the readiness of the context may allow implementation to be more successful.

Objective: This study presents results from adaptation of the Organizational Readiness for Implementation Change
(ORIC) instrument to assess educators' readiness to implement the nutrition education curriculum Together, We Inspire Smart Eating (WISE). This study presents psychometric properties of the ORIC in this new setting and assessment of association with perception of barriers.

Study Design, Setting, Participants, Intervention: Educators and administrators $(\mathrm{N}=359)$ completed the ORIC before receiving education on the implementation of WISE. Participants were primarily lead teachers (45\%) with at least some college education (64\%).

Outcome Measures and Analysis: The ORIC instrument ( $\mathrm{N}=12$ items rated on 5-point likert scale) has demonstrated validity and reliability in health care settings. Participants were asked to rate nine potential barriers to implementation.

Results: The reliability for the 12 items was $(\alpha=.97)$. An exploratory factor analyses $(E F A$, eigenvalue $=1$ ) indicated one factor. An EFA constrained to replicate previous work with two factors was not supported. Examination of careless response include split half reliability $(\alpha=.92)$ and an examination of the number of repeating responses (34\% used the same response for all items) suggested respondents did not distinguish items uniquely. However, the validity of the total ORIC was supported by the expected association with the number of barriers perceived by participants $(\mathrm{r}=-30)$.

Conclusions and Implications: The ORIC had high internal consistency and demonstrated convergent validity in this sample. However, indicators of careless responding were elevated suggesting that the ORIC may benefit from a reduction of items. Alternately, it may be possible to present the items in a format that emphasizes the unique contribution of each question.

Funding: NIH, FNS.

\section{P101 Reliability of the Self-Report Home Food Inventory and the Food Preparation Supplies Checklist in College Students}

Abeer Almudaihim, MS, University of Florida; Anne Mathews, PhD, RDN; Melissa Vilaro, PhD; Sarah Colby, PhD, RDN, University of Tennessee; Karla Shelnutt,PhD, RDN, kpagan@ufl.edu, University of Florida, 3028 McCarty Hall D, Gainesville, FL 32611

Background (Background, Rationale, Prior Research, and/or Theory): The home food environment (HFE; food availability and food preparation supplies) appears to be an important mediator of diet quality. The Home Food Inventory (HFI) and the Food Preparation Checklist (FPC) are commonly used tools for assessing HFE but have only been tested via in-home researcher visits, which are time consuming and expensive, but have not been tested via self-report in college students.

Objective: To evaluate the reliability of college students' self-reported HFE using the HFI and the FPC.

Continued on page 556 